\title{
Technology and computing for the sake of the physician
}

\author{
The abstracts of the 26th congress of ESCTAIC, Timisoara, Romania, September 22-24 \\ 2016
}

\author{
Gabriel M. Gurman ${ }^{1}$
}

Published online: 24 February 2017

(C) Springer Science+Business Media Dordrecht 2017

Once again we acknowledge the opportunity offered to the European Society of Technology and Computing in Anesthesia and Intensive Care (ESCTAIC) to publish some of the abstracts of the lectures presented in the framework of its last congress, held for a third consecutive time in the nice and old city of Timisoara, Romania.

A large number of speakers from 11 countries contributed to the scientific program. Both plenary lectures have been dedicated to the subject of physics for the clinician. Two American physicists, Drs Yakov Pipman and Marco Zaider, presented various aspects of physical principles applicable in daily practice as well as some professional hazards related to radiation exposure.

The tradition of dealing with the ultrasound use in anesthesia and critical care was preserved and this year a workshop on the place of ultrasound in airway management was organized by Dr. Daniela Godoroja (Romania) and attended by young specialists and residents in Anesthesiology.

This year the scientific committee decided to dedicate a good part of the program to new technological achievements contribution the physician's daily activity. Some new technological aspects in our routine work are very well known but less understood. Other methods and techniques are still not used in all corners of the world, in all operating rooms and intensive care units.

There is also no secret that we, physicians, in our continuous activity for improving our patients' chance to heal, too often forget to pay attention to the working place reality and to try to improve our working conditions.

Gabriel M. Gurman

gurman@bgu.ac.il

1 Mayney Hayeshuah Medical Center, Ben Gurion University of the Negev, Bnei Brak, Israel
Sometime we do not know how to deal with new equipment, and many times we are overwhelmed by the amount of work and do not succeed in organizing our own activity, in order to overcome the difficulties imposed by a too strong production pressure.

The 2016 Timisoara congress tried to give an answer to all the above problems.

The first of the eleven panels dealt with the problem of acute postoperative pain and practical solutions offered to the clinician for overcoming the high percentage of surgical people who complain of severe pain after operation. Neamtu discussed the persistent postsurgical pain [1]. This condition, which significantly increase the anesthesiologist's workload, is mostly associated with limb amputation, thoracotomy, mastectomy, coronary bypass graft, inguinal hernia repair, caesarian section, etc. The author recommends a multimodal approach, including regional anesthesia, drug affecting central sensitization (like ketamine, gabapentin) and a careful immediate postoperative analgesia.

A second lecture discussed the role of the ultrasound technique in performing nerve blocks [2]. Onutu emphasized the role of this technique in increasing the rate of success, by increasing the precision and accuracy of the method, beside the intrinsic advantages for the patient, such as reduction in total dose of anesthetic and avoidance of complications.

The last lecture of the panel presented the impact of preoperative anxiety on the incidence and level of postoperative pain [3]. Gurman reported new data which are supposed to convince the clinician about the importance of preventing the postoperative pain by a good psychological preoperative preparation of the patient and the use, if necessary, of antidepressant drugs before surgery. His lecture was based on some studies which show the positive impact 
of this preparation on the magnitude and frequency of postoperative pain.

The immediate postoperative pain prevention and treatment was the subject of another panel, focused on pediatric patients.

Beltran, from the National Children's Hospital, Columbus, USA, presented two papers, the first one [4] about management of pain after surgery for pectus excavates (the Nuss procedure, which is a minimally invasive thoracoscopic technique used to repair pectus excavatum or carinatum using placement of a metal bar in the thoracic cavity). The author proposed a combined technique of thoracic epidural analgesia and continuous infusion of local anesthetics and opoids, as part of the well known concept of multimodal approach in the treatment of pain. The same idea, of continuous i-v infusion of local anesthetics as a mean of preventing postoperative pain, was presented in Beltran's second paper [5]. The proposed solution discussed the possibility to continue the infusion in the ambulatory setup, by a strong cooperation with the patient's family and the availability of experts in regional analgesia around the clock.

Fiocca [6] presented in the same panel a very interesting summary of various anatomical approaches for locoregional anesthesia in maxillo-facial surgery. The speaker presented the technique of anesthetizing the maxillary nerve, the infraorbital block for lip surgery and the palatine nerves block for palate surgery. Most of the slides were tridimensional, and this fact offered the audience clear details of the anatomy of the face.

Another panel suggested that technical innovations in the anesthesiologist's environment could improve his/her working conditions and performance.

The opening lecture [7], presented by Czaplik, a medical technology expert, offered the clinicians some thoughts and practical advises regarding the use of smartphone in the daily activity. Special applications would serve for calculating drug doses, getting access to the drug database, accessing medical records and obtaining online support for reaching a correct clinical decision.

A better efficiency in the operating room (OR) would contribute to the reduction of the production pressure and also would decrease the level of the professional stress of the anesthesiologist. A lecture on the impact of OR efficiency on human activity was the subject of a presentation coming from Italy [8]. Corso presented a project coming from GB Morgani L Pierantoni Hospital in Forli, which includes a data recording system of the surgical process of every patient. The system creates a practical and easy- touse data, which gives the managers the needed information for increasing the OR efficiency by improving the schedule and also by assuring the patient safety.

Rauch [9] discussed the new aspects of using extracorporeal $\mathrm{CO}_{2}$ removal in the treatment of ARDS. He presented a project of a multicenter study which aim is to investigate the efficiency of a combining low tidal volume with the use of an ultra-flow pumpdriven device. This device is supposed to provide removal of up to $50 \%$ of the basal $\mathrm{CO}_{2}$ production at a low flow of not more than $500 \mathrm{ml} / \mathrm{min}$.

Two lectures addressed the topic of the role of simulation in training. In many countries simulation centers are just a beginning and Dirzu and Copotoiu [10] tried to oppose the idea that too much money is invested in this kind of activity, by explaining that simulation as part of a training process, could prevent malpractice accusations and improve the physician's skills. Bryant [11] discussed the place of simulation in the OR environment for teaching and learning the technique of fiberoptic intubation. The report presented the use of a bronchoscopy simulator, used directly prior to actual patient care.

Venema and coauthors [12] presented a poster about photoplethysmography imaging, which may bring two essential advantages for the ICU: non-contact, hence applicable to wounded skin regions and spatial resolved vital parameter monitoring. Their work analysed the physical limitation of spatial resolved monitoring of $\mathrm{S}_{\mathrm{p}} \mathrm{O}_{2}$ with Monte Carlo simulation.

The subject of quantification of the surgical stress seems to be unsolved, yet. This topic is important for the anesthesiologist, since its magnitude has an impact on the level of depth of anesthesia in each surgical procedure and also determines the probable degree of postoperative pain. A special panel was dedicated to this subject. Gurman [13] described the pathophysiology of the surgical stress and the various, and not too successfully, trials to quantify surgical stress. Biro [14] reported the results of a multicenter trial in which a special score was used for various surgical interventions in order to validate it. The variables of the proposed score, called Preliminary Universal Surgical Invasiveness Score ( $p U S I S)$, included size of incision, anatomic location of surgical intervention, the target organ, need for blood replacement and need for surgical site drainage. The results of the first 80 cases proved the feasibility of the score, but more data are needed in order to get a final conclusion.

In the framework of a panel entitled "How to be sure you did not give too much or too little" Brull [15] discussed the need for an adequate level of neuromuscular block, mainly when the surgical procedure demands a better exposure and a deeper muscle relaxation This approach would not only facilitate the surgeon's work but also would preserve the hemodynamic stability. With the introduction of sugammadex, a quick recovery is a desiderate easy to achieve.

Margason [16] presented the use of a new device for nasal administration of high flow oxygen. After discussing its main areas of use, the author concluded that this therapy 
could change the nature of difficult intubation, by enlarging the "apneic window" and thus preserving the safety of the technique.

A large place in the scientific program was allocated to the various aspects of the anesthesiologist's work in special, difficult environments.

For example, Dirzu [17] reported the results of a preliminary trial of using a new device for intranasal administration of midazolam in premedication. In comparison to the "classical" devices this new apparatus delivers a sufficient large dose of drug in one single push on the button. It could be easily used in cases there is no i-v route open or in children with a low degree of cooperation.

Belii and coauthors [18] proposed a new software for managing the data on chronic medication, which could interest the anesthesiologist and the surgeon during the perioperative period. The proposed system would offer the clinician more information and also would give a chance to avoid errors and drug side effects.

Syed [19] discussed in his lecture the implications of the mass-destruction weapons warfare on the physician perspective. The presentation covered both situations, of war and also terrorist attacks, when the use of nuclear, biological or chemical weapons could affect large masses of military and civilians. Beside the advices concerning the first aid, the lecture discussed the preparation of hospitals and operating rooms in order to be able to treat a large variety of casualties.

Turchetta is an Italian specialist in Anesthesiology who often accompanies the Italian Red Cross Army in its campaigns abroad. During long periods of time spent in countries in war or in regions with a very modest capability to manage diseases and trauma, he accumulated a large experience as an anesthesiologist. He is often asked to innovate and improvise in order to offer his patients proper treatment. In his lecture [20] Turchetta presented a series of clinical scenarios regarding management of mass casualties and also some cases or which he was obliged to find unusual solutions. He also described the human difficulties encountered during conflicts and the absolute need to periodically organize field drills, combined with assessment of the staff ability to cope with difficult situations.

Ohligs and his colleagues [21] prepared a poster presentation on the use of telemedicine in case of mass casualties. Their proposal included a series of software programs, which would facilitate patient management in case of multiple casualties, among them one which would help in classifying the patients and also the results of treatment.
The large variety of the subjects assured a full-hall audience and very interesting discussions.

We do hope that the concept of assisting the clinician in his/her daily activity by improving the understanding of new technology and its use would be present in our future meetings, too.

We also hope that the Editorial Board of JCMC will find the included presentations as fit for publishing in full text and we are deeply grateful for getting the opportunity to present in short some important parts of our scientific program in this very esteemed Journal.

\section{References}

1. Neamtu A. Persistent postsurgical and postinjury pain-un apdate.

2. Onutu AH. Ultrasound guided nerve blocks: that makes us happy?.

3. Gurman GM. A message to your patient: if he/she is too anxious before surgery, there would be more pain in the postoperative period.

4. Beltran R. NUSS procedure: clinical options for postoperative pain management and their side effects.

5. Beltran R. Regional anesthesia and ambulatory surgery: the role of continuous infusion devices in postoperative pain management in pediatrics.

6. Federico Fiocca. Locoregional blocks in maxilla-facial surgery. Advises for the anesthesiologist.

7. Czaplik M. Medical apps: potentials and risks for the anesthesiologist.

8. Corso RM Operating room efficiency: technology and human factors.

9. Rauch S. New technology in extracorporeal $\mathrm{CO}_{2}$ removal.

10. Dirzu DS, Copotoiu S. The role of simulation in medical education.

11. Bryant JA. Use of simulation for resident training.

12. Boudewijn Venema et al. Pulse Oximetry Imaging-Practical Feasibility and Theoretical Limitations.

13. Gurman GM Surgical trauma and its influence on anesthesia technique.

14. Biro P What is USIS? It is useful? And what can it do?

15. Brull SJ. Deep neuromuscular block: when do I pay for my sins?.

16. Margason M. Nasal high flow oxygen therapy. What the anaesthetist should know.

17. Dirzu $\mathrm{D}$ et al. New generation nasal delivery device for preoperative sedation-safe, effective and physician's choice.

18. Belii A et al. Assisted by a simple software, the perioperative management of chronic medication improves patient safety and easies the physician activity.

19. Syed A. Nuclear, biological and chemical warfare: a healthcare provider's perspective.

20. Turchetta B. Major difficulties in prividin anesthesia in mass casualties-how to prevent them by practical exercise.

21. Ohligs $\mathbf{M}$ et al. Mass casualty incident management supported by augmented reality and telemedicine. 